\title{
Order and Frustration in Artificial Magnetic Patterns
}

\author{
H. Zabel, A. Schumann, A. Westphalen and A. Remhof \\ Department of Physics and Astronomy, Ruhr-Universität Bochum \\ Universitätstr. 150, 44780 Bochum, Germany
}

\begin{abstract}
Artificial magnetic dipole arrays arranged on a square lattice exhibit a fascinating variety and complexity of configurations. Among the 16 possible configurations, six fulfill the spin ice rule of two dipoles pointing into a vertex and two point out. We present experimental realizations of magnetic dipole arrays and discuss the remanent state as well as the magnetization reversal in an external field.
\end{abstract}

PACS numbers: $75.75 .+\mathrm{a}, 75.60 . \mathrm{Jk}, 41.20 . \mathrm{Gz}$

\section{Introduction}

Modern lithographic techniques enable the fabrication of lateral magnetic patterns of various sizes, shapes, and periodicities on a nanometer to micrometer scale [1,2]. Of particular interest are artificial magnetic dipoles, which can be arranged on lattices with a variety of symmetries [3, 4]. Some arrangements mimic parallel or ferromagnetic order, others antiparallel or antiferromagnetic order [5-7]. More complex triangular and square lattices are also feasible, where the dipoles are either arranged on any of the vertices or between the vertex points. In the latter case, three or four magnetic dipoles meet in any one vertex, which automatically leads to different degrees of frustration. Recently, the ground state and the magnetization reversal has been studied for square lattices [8-10], honeycomb lattices [11], and kagomé lattices [12]. In this contribution we concentrate on dipolar arrays placed on square lattices and study different configurations, correlation effects, and the magnetization reversal within the sublattices.

\section{Sample preparation}

The aim was to fabricate magnetic dipoles, which exhibit their dipolar character by the shape and the aspect ratio rather than by the crystal anisotropy. Therefore we have chosen magnetically soft polycrystalline permalloy $\left(\mathrm{Py}=\mathrm{Ni}_{0.8} \mathrm{Fe}_{0.2}\right)$ as magnetic material. A homogeneous $25 \mathrm{~nm}$ thick Py film was first deposited onto a polished $\mathrm{Si}$ substrate by ion-beam sputtering. Subsequently, the film was spin coated with a negative tone resist and the pattern was defined by e-beam lithography using a modified Quanta 200 FEG scanning electron microscope equipped with a Raith Quanta lithography unit. After e-beam exposure and developing, the structures were transferred into the metallic film by ion beam etching. The aspect ratio of 10:1 (length:width) for the dipoles was kept constant and promises a single domain state with a high remanence and with a clear dipolar character, according to the phase diagram for magnetic bars [13]. The distance between the dipoles was varied from $0.4 \mu \mathrm{m}$ to $3.6 \mu \mathrm{m}$ in order to alter the dipolar interaction. Scanning electron micrographs (SEM) of some patterns are shown in Fig. 1. Let us note that the three individual micrographs were recorded with different magnifications, a marker showing the respective length scale has been added to each image. Magnetic force microscopy (MFM) images of the sample confirm the single domain state of the individual islands in remanence, as shown in Ref. $[10,14]$. The MFM images (Nanoscope IIIa, Digital Intruments) were recorded at remanence and at room temperature after saturating the patterns in well defined directions.

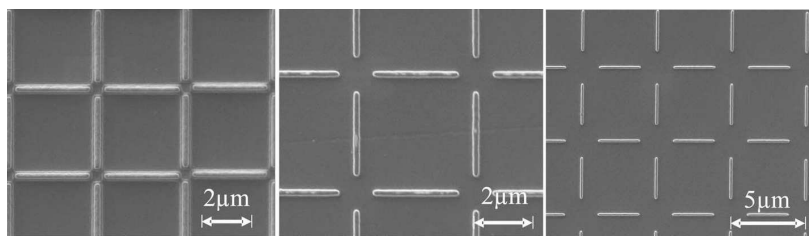

Fig. 1. SEM micrographs of rectangular permalloy bars placed on a square lattice with separation of $0.42 \mu \mathrm{m}, 0.84 \mu \mathrm{m}$ and $1.68 \mu \mathrm{m}$ distances (left to right). In all patterns the bars have the same size, only their separation differs. Figure from Ref. [10].

\section{Magnetic configurations}

Magnetic dipoles on a square lattice can be characterized according to different schemes. One possible characterization is the counting of domain walls that are present in a square and considering their location. Using this scheme, we distinguish between vortex states with zero domain walls, onion states with two domain walls positioned at the diagonal of the squares, and horseshoe states with two domain walls on the same horizontal or vertical line. In Fig. 2 the vortex state is designated as type I, onion states are designated as type II F and 


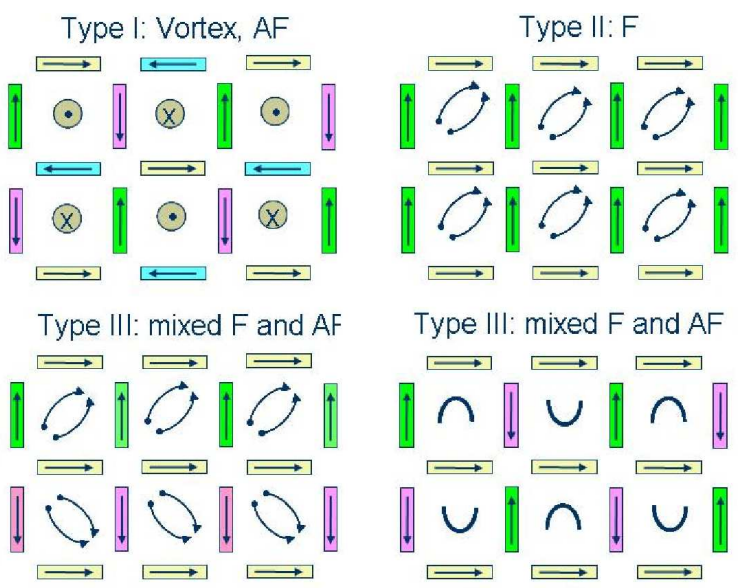

Fig. 2. Magnetic dipoles arranged on a square lattice. Some possible orders are shown, including a periodic array of alternating vortices and antivortices, ferromagnetic order in the horizontal and vertical sublattices, ferromagnetic order in the horizontal sublattice but antiferromagnetic order in the vertical sublattice. The position of domain walls is indicated by arrows and half circles within the squares. The vortex state has zero domain walls, the onion state has domain walls across the diagonal of a square on the upper right and lower left corner, and the horseshoe state has two domain walls within the horizontal or vertical sublattices.

type III mixed F and AF, and the horseshoe state is labelled as type III mixed $\mathrm{F}$ and $\mathrm{AF}$.

Another possibility is to consider the symmetry of the magnetic order (see Fig. 2). We can distinguish between ferromagnetic order along the horizontal and the vertical bars (type II F), mixed ferromagnetic order in the horizontal bars and antiferromagnetic order in the vertical bars (type II mixed and type III mixed), or antiferromagnetic order parallel to the horizontal and to the vertical bars (type I). This latter configuration forms a microvortex state of alternating chirality, which may also be called an antiferromagnetic toroidal structure.

The most transparent characterization of possible configurations in the dipolar square lattice is the counting of the number of dipoles pointing into a vertex versus those pointing out of a vertex. This is shown in Fig. 3. In the type I configuration two dipoles point in, and two point out, yielding a zero effective magnetic moment. If each dipole represents a positive charge for pointing in and a negative charge for pointing out, the sum of charges is zero. There are only two configurations which fulfill these conditions. In type II as well as in type I two dipoles point in and two point out. Therefore again the charge is zero. However, since the dipoles pointing out are on the same corner instead of being on opposite corners, there is a resulting magnetic moment directed along the diagonal indicated by a dashed arrow in Fig. 3 . A total of 4 configurations are available with this symmetry. Type III configurations contain 8 variations with three dipoles pointing into a vertex and one pointing out, yielding a charge of \pm 2 and an effective dipole moment in either the horizontal or the vertical direction. Finally in type IV four dipoles either point in or out, resulting in a charge \pm 4 and no magnetic moment. All together a total number of 16 configurations can be realized in the square lattice.

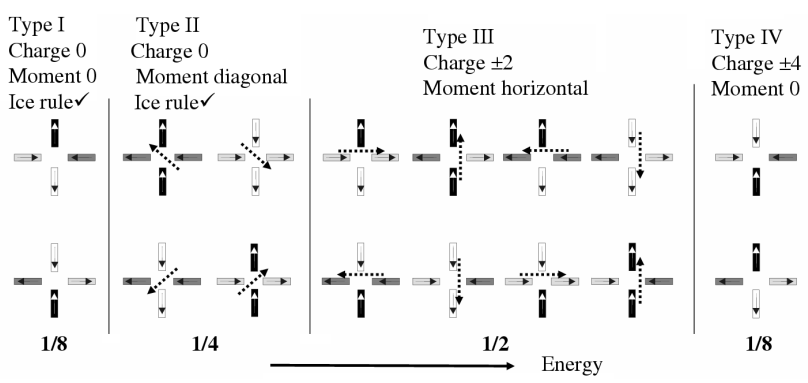

Fig. 3. Different configurations can be categorized according to the number of magnetic dipoles pointing into a vertex versus the number of dipoles pointing out. Assigning a charge to the poles of a dipole, the sum of charges in type I and type II vertices adds to zero, but type II has a finite effective magnetic moment parallel to the diagonal of a vertex. Type III vertices have a charge of \pm 2 and an effective moment parallel to one of the sublattices. Type IV has a charge of \pm 4 but no residual magnetic moment. The gray shades of the dipoles follow the scheme: black $=u p$, white $=$ down, light gray $=$ right, dark gray $=$ left.

If the configurations in a square lattice were statistically distributed, we expect to find type I and type IV configurations with a probability of $12.5 \%$, type II configurations with a probability of $25 \%$, and type III configurations with a probability of $50 \%$. This purely statistical argument only applies as long as energy and correlation effects can be neglected.

Let us first consider correlation effects. On the average the sum over all charges should be zero [15]. Creating a configuration of type III with charge +2 will automatically require the formation of a configuration with charge -2 . The creation of a type IV vertex can be compensated by two type III vertices of opposite charge. Therefore, the creation and annihilation of type III and type IV vertices is highly correlated. Type I and II are neutral and therefore the creation and annihilation are uncorrelated. Furthermore, type I and II fulfill the so-called ice rule. In the ice structure, two hydrogen atoms are close to the oxygen atoms and two are further away. This rule is usually quoted as the "two-in-two-out" ice rule. Figuratively speaking, the magnetic dipoles on the square lattice obey the spin-ice rule, if two spins or magnetic dipoles point into a vertex and two point out [16]. In this respect, vertices of type I and II belong to the spin ice case. They differ only with respect to their effective magnetic moment. Type III and IV vertices violate the spin-ice rule.

From an energy point of view the energy increases from type I to type IV vertices [15]. Type I has the lowest en- 


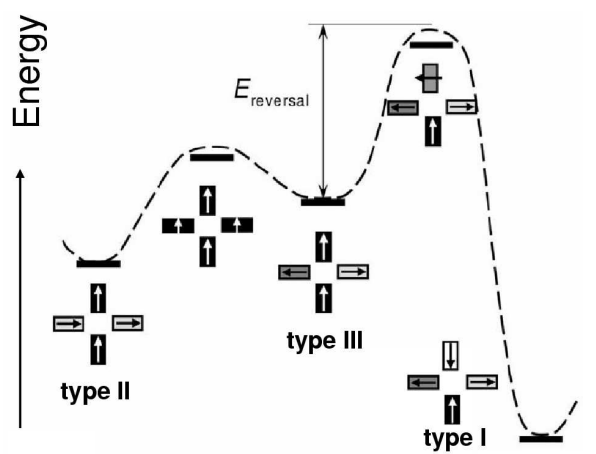

Fig. 4. The energy landscape for different configurations is shown together with the energy required to reverse a dipole for a transition from one configuration to another. The gray shades of the dipoles follow the same scheme as in Fig. 3. Figure from Ref. [10].

ergy as two dipoles opposing each other have the largest distance. In type II, opposing dipoles share one corner, such that there is an increased magnetostatic repulsion. Type III vertices have a higher energy than type II vertices because of magnetostatic reasons. The energy landscape for the different vertices is shown in Fig. $4[4,10]$. The maxima between the minima are due to the shape anisotropy, which has to be overcome for the transition from one type to another. The maximum is particularly high for the transition from type III to type I as one dipole has to be reversed passing through the hard magnetic axis, while all other dipoles remain constant. Therefore without a local field that specifically selects certain dipoles to reverse, the transition from type III to type I is rather unlikely.

We have tested experimentally some of the predictions for dipolar square lattices, which is discussed in the next section.

\section{The remanent magnetization state}

We have investigated several patterns of magnetic dipoles on square lattices with different separations between the dipoles. For imaging the remanent state we used MFM. Different paths to the remanent state can be taken and subsequentially imaged. An external magnetic field can be applied either parallel to one of the sublattices or along the diagonal of the squares. Or the pattern can be demagnetized in a rotating external field with diminishing field amplitude [9]. The most straight forward magnetization path is the one along the diagonal. This will favor all configurations with an effective magnetic dipole directed in the diagonal of a vertex, i.e. in the remanent state it will favor the type II configuration over all other possible configurations. Indeed, this is also observed in the experiment [10].

Magnetization parallel to one of the two sublattices will leave freedom of orientation for the perpendicular sublattice when returning from saturation to remanence. Thus we expect a certain randomness in the dipolar orientation within the sublattice perpendicular to the applied field. We have tested this conjecture for square lattices with different dipolar separations. For the largest separation of $3.4 \mu \mathrm{m}$ we indeed find a mixture of type II and type III vertices as shown in Fig. 5. The relative occurrence of type II and type III is $2: 1$, which is exactly expected from their energy difference $[4,10]$.

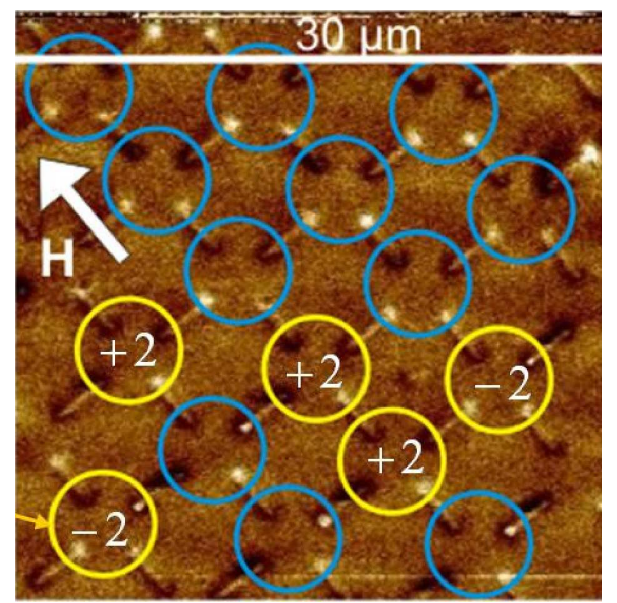

Fig. 5. Magnetic force microscopy image of a square lattice of magnetic dipoles shown in remanence after saturation along the field direction indicated by the white arrow. Different types of vertex configurations are found: blue circles indicate type II vertices, yellow circles indicate type III vertices. The numbers in the circles is the magnetic charge of the particular vertex. For further details, please refer to the main text.

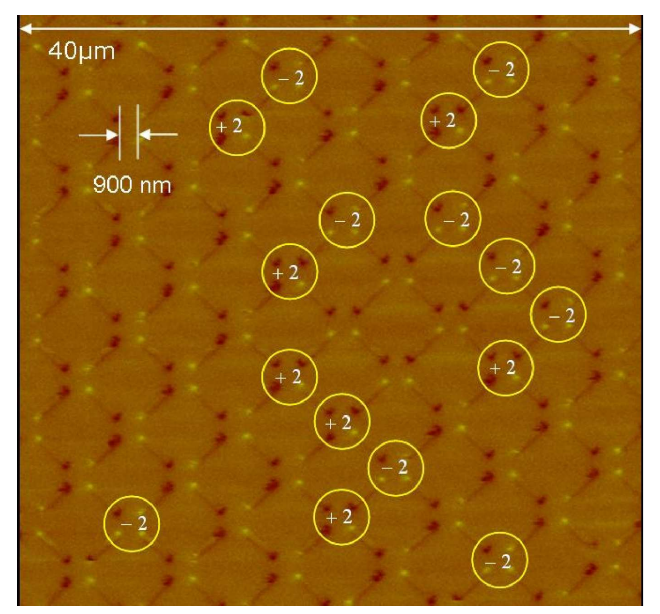

Fig. 6. Magnetic force microscopy image of a square lattice of magnetic dipoles shown after growth and lithographic steps and before exposure to a magnetic field in the virgin state. Only type III vertices are marked by circles and the magnetic charge is indicated inside of the circles.

Of interest is also the virgin state, i.e. the state immediately after sample preparation and without exposure to 
an external field. In Fig. 6 the virgin state of a larger array containing 55 vertices is shown. They subdivide into 39 vertices of type II with zero charge and 16 vertices of type III with charge \pm 2 . There are eight type III vertices with charge +2 and eight with charge -2 , marked by circles. Thus the total charge is zero as expected. The ratio of type II to type III vertices is $2.5: 1$, as compared to a ratio of 1:2 expected for a statistical average. However, it is close to the 2:1 ratio expected from the energy landscape of the vertex configurations. This shows that even in the virgin state of the present pattern correlation effects cannot be neglected. For a statistical average either the pattern should be completely demagnetized in an external rotating field [9], or the dipoles should be further separated.

\section{Magnetization reversal}

In a complex pattern such as the square dipolar lattice the magnetization reversal of the individual horizontal and vertical sublattices are difficult to study separately. Imaging with MFM might be a solution if the magnetic tip is stable enough in the external field. Photoemission electron microscopy (PEEM) can only be used in small fields. Only magnetic transmission X-ray microscopy (MTXM), Kerr microscopy (KM), and Bragg magneto-optical Kerr effect (Bragg-MOKE) remains. We have chosen Bragg-MOKE, which is, however, not an imaging technique in real space, but in the Fourier space [17-19]. With Bragg-MOKE the magnetic hystereses for different orders of interference are determined, which yields the Fourier components of the magnetization distribution within the bars, i.e. the magnetic form factor. Using a micromagnetic simulation package such as OOMMF [20] the magnetization distribution in the bars can be calculated for different field values and the magnetic hysteresis of different orders is extracted. If one finds good agreement between the measured and calculated hysteresis curves from specular MOKE up to higher order of interference, the simulated magnetization distribution has a high level of confidence to reflect the correct magnetization distribution. We have used this method to study the magnetization distribution of a square lattice of similar dimensions as the ones discussed before, but using $\mathrm{Fe}$ instead of Py for better Kerr sensitivity. For the micromagnetic simulations we used the following parameters: exchange stiffness $21 \times 10^{-13} \mathrm{~J} / \mathrm{m}$, saturation magnetization $500 \times 10^{3} \mathrm{~A} / \mathrm{m}$, and the anisotropy constant $K_{1}=0$. The mesh cell size was chosen to be $15 \mathrm{~nm}$.

In Fig. 7 we show micromagnetic simulations for a square lattice with a separation of the bars in the vertices of $1.9 \mu \mathrm{m}$. The magnetic field is applied parallel to the horizontal sublattice. The micromagnetic simulation depicts only a small section of the whole structure. Starting from negative saturation, the horizontal islands maintain their dipolar orientation parallel to the negative field direction up to the coercive field of about +100 Oe. Surprisingly, the magnetization in all vertical islands rotate to the down direction upon decreasing the field, such

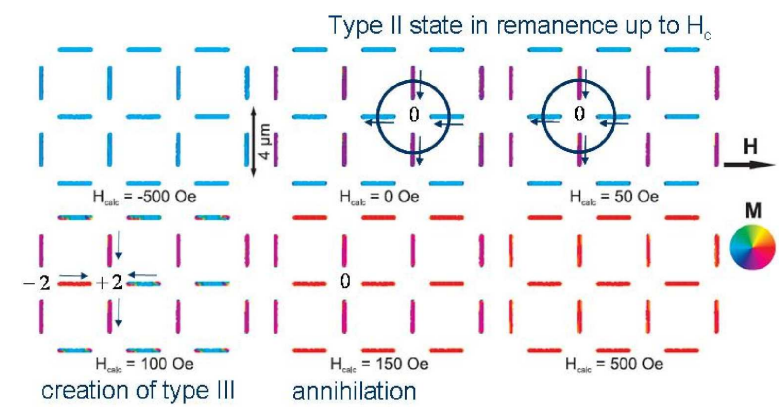

Fig. 7. The magnetization reversal of a square lattice is shown for different field values. The magnetization distribution shown is the result of micromagnetic simulations using the OOMMF code and adapted to Bragg-MOKE measurements of square lattices.

that at remanence up to the coercive field an ordered ferromagnetic array in both the horizontal and the vertical islands is present. This configuration can be characterized as an onion state or a type II state, which fulfills the ice rule. Further increase in the magnetic field beyond the coercive field forces the horizontal islands to reverse their magnetization, most likely via a domain wall process. However, as there is a slight distribution of switching fields, some horizontal island have already reversed their magnetization at +100 Oe, while others have not. This leads to the creation of pairs of type III vertices with charge \pm 2 , such that the total charge remains zero. After all horizontal islands have reversed their magnetization direction $(+150 \mathrm{Oe})$, again a type II onion state is established, however with the domain walls in each square moved from the upper right corner and bottom left corner to the upper left corner and bottom right corner. Thus between the coercive fields the ferromagnetic alignment of the horizontal islands can be switched without affecting the magnetization of the vertical islands. The same would also apply if the magnetic field is applied in the vertical direction. Therefore in this pattern the orthogonal sublattices can be addressed individually by applying an external field either parallel to one or the other sublattices. This is only possible if the islands have a sufficient separation. Patterns with closer spacing of the islands show considerable interaction via magnetostatic fields.

\section{Summary}

In summary, we have discussed different magnetic ordering of micromagnetic dipole arrays arranged on square lattices. A total of 16 vertex configurations can be distinguished, which subdivide further into four types, according to their effective magnetic charge and magnetic dipole moment. Only the first two types fulfill the ice rule, i.e. two dipoles point into a vertex and two point out. Type II configurations are the most commonly observed ones and they build the basic unit for ferromagnetic order in both sublattices. However, for sufficient separation between the magnetic islands, type III configurations can be real- 
ized in the remanent state. By flipping one dipole within a type II pattern, two type III configurations are created with opposite charge, such that charge neutrality is conserved. MFM images of the remanent state and Bragg-MOKE measurements of the magnetization reversal confirm the basic rules and the ratio of $2: 1$ between type II and type III configurations either in the virgin state or in the remanent state after saturation parallel to one of the sublattices in case that the magnetostatic interaction is weak. Type III configurations can be found locally, but large ordered patterns of type III configurations have not been found yet. Type I with a microvortex pattern of alternating chirality corresponds to the ground state of the system. Due to the large energy barrier which has to be overcome to switch the system from a type III into a type I state, this configuration has not been observed. Type IV is unlikely to be realized because of the large magnetostatic repulsion.

Concluding, magnetic dipole arrays on square lattices offer a large variety of configurations and magnetic orders. We are just at the beginning to explore their complex hierarchical order and correlations and the phase transitions between them.

\section{Acknowledgments}

We would like to thank the DFG for financial support within the framework of the collaborative research centers SFB 491 Magnetic Heterostructures: Spin Structure and Spin Transport.

\section{References}

[1] J.I. Martín, J. Nogués, K. Liu, J.L. Vicent, I.K. Schuller, J. Magn. Magn. Mater. 256, 449 (2003).

[2] C.L. Dennis, R.P. Borges, L.D. Buda, U. Ebels, J.F. Gregg, M. Hehn, E. Jouguelet, K. Ounadjela, I. Petej, I.L. Prejbeanu, M.J. Thornton, J. Phys., Condens. Matter 14, R1175 (2002).

[3] E.Y. Vedmedenko, Competing Interactions and Pattern Formation in Nanoworld, Wiley, Weinheim 2007.

[4] E.Y. Vedmedenko, N. Mikuszeit, Chem. Phys. Chem. 9, 1222 (2008).
[5] K. Temst, M.J. Van Bael, J. Swerts, H. Loosvelt, E. Popova, D. Buntinx, J. Bekaert, C. Van Haesendonck, Y. Bruynseraede, R. Jonckheere, H. Fritzsche, Superlattices Microstruct. 34, 87 (2003).

[6] A. Remhof, C. Bircan, A. Westphalen, J. Grabis, A. Nefedov, H. Zabel, Superlattices Microstruct. 37, 353 (2005).

[7] A. Westphalen, K. Theis-Bröhl, H. Zabel, K. Rott, H. Brückl, J. Magn. Magn. Mater. 302, 181 (2006).

[8] R. Wang, C. Nisoli, R.S. Freitas, J. Li, W. McConville, B. Cooley, M.S. Lund, N. Samarth, C. Leighton, V.H. Crespi, P. Schiffer, Nature 439, 303 (2006).

[9] R.F. Wang, J. Li, W. McConville, C. Nisoli, X. Ke, J.W. Freeland, M. Grimsditch, P. Lammert, V.H. Crespi, P. Schiffer, J. Appl. Phys. 101, 09J104 (2007).

[10] A. Remhof, A. Schumann, A. Westphalen, H. Zabel, N. Mikuszeit, E.Y. Vedmedenko, T. Last, U. Kunze, Phys. Rev. B 77, 134409 (2008).

[11] Yi Qi, T. Brintlinger, John Cumings, Phys. Rev. B 77, 094418 (2008).

[12] A. Westphalen, A. Schumann, A. Remhof, H. Zabel, M. Karolak, B. Baxevanis, E.Y. Vedmedenko, T. Last, U. Kunze, T. Eimüller, Phys. Rev. B 77, 174407 (2008).

[13] T. Last, S. Hacia, M. Wahle, S.F. Fischer, U. Kunze, J. Appl. Phys. 96, 6706 (2004).

[14] A. Remhof, A. Schumann, A. Westphalen, T. Last, U. Kunze, H. Zabel, J. Magn. Magn. Mater. 310, 794 (2007).

[15] G. Möller, R. Moessner, Phys. Rev. Lett. 96, 237202 (2006).

[16] S.T. Bramwell, Nature 439, 273 (2006).

[17] M. Grimsditch, P. Vavassori, J. Phys., Condens. Matter 16, R275 (2004)

[18] A. Westphalen, M.-S. Lee, A. Remhof, H. Zabel, Rev. Sci. Instrum. 78, 121301 (2007).

[19] W. Kleemann, Rev. Sci. Instrum. 78, 120901 (2007).

[20] M.J. Donahue, D.G. Porter, Interagency Report NISTIR 6376, National Institute of Standards and Technology, Gaithersburg, MD (Sept. 1999). 